\title{
Demographic, Socio-Economic and Psychosocial Determinants of Current and Consistent Condom Use among Adolescents in Botswana
}

\author{
Enock Ngome1,2 \\ ${ }^{1}$ Department of Population Studies, Faculty of Social Sciences, University of Botswana, Gaborone, Botswana \\ ${ }^{2}$ Demography and Population Studies Program, University of the Witwatersrand, Johannesburg, South Africa \\ Email: enock.ngome@gmail.com
}

How to cite this paper: Ngome, E. (2016) Demographic, Socio-Economic and Psychosocial Determinants of Current and Consistent Condom Use among Adolescents in Botswana. World Journal of AIDS, 6, 137-156.

http://dx.doi.org/10.4236/wja.2016.64017

Received: September 19, 2016

Accepted: November 7, 2016

Published: November 11, 2016

Copyright $\odot 2016$ by author and Scientific Research Publishing Inc. This work is licensed under the Creative Commons Attribution International License (CC BY 4.0).

http://creativecommons.org/licenses/by/4.0/ (c) (i) Open Access

\begin{abstract}
Background: To facilitate effective program designs aimed at assisting youth avoid unnecessary negative reproductive health outcomes, there is need to understand determinants of condom use among adolescents. Objectives: To determine demographic, socioeconomic and psychosocial factors associated with adolescent condom use in Botswana. Methods: Logistic regression models were employed to analyse a weighted sample of 221 adolescents aged 15 to 19 years from the 2012 Botswana AIDS Impact Survey who had a sexual relationship during the 12 months preceding the survey. Results: A majority of adolescents were current and consistent condom users ( $81 \%$ and $72 \%$ respectively). Both current and consistent condom use were positively associated with secondary or higher education, unemployed, high perception of severity of HIV to health and high self-efficacy perception. However, being female, non-Christian, having medium or comprehensive HIV/AIDS related knowledge and high perceived personal risk to HIV infection were negatively associated with both outcomes. Although being 18 to 19 years, having multiple sexual partners and high perception of condom attributes were positively associated with current condom use, they were negatively associated with consistent use. Conclusions: The study showed that in addition to demographic and socioeconomic factors, psychosocial factors are important predictors for condom use among adolescents.
\end{abstract}

\section{Keywords}

Adolescents, Condom Use, Psychosocial, Botswana

\section{Introduction}

Risky sexual behaviour among adolescents remains one of the emerging public health 
concerns globally. Compared to developed countries, there has been an apprehension on the increasing number of sexually active adolescents in developing countries, more especially the Sub-Saharan Africa (SSA). Adolescents in several of these developing countries, including Botswana experience early sexual debut [1] which is usually unplanned and as a result, many of the adolescents become victims of several negative sexual and reproductive health outcomes such as HIV infection, sexually transmitted diseases (STDs), unplanned teenage pregnancies and unsafe abortions. The use of condoms during all sexual encounters by adolescents has been identified as one of the major interventions aimed at reducing these reproductive health problems.

Botswana has one of the highest HIV prevalence rates in the world [2]. The incidence rate among adolescents aged between 15 and 19 years in Botswana is estimated at $0.7 \%$. The proportion of teenage women who have ever been pregnant stands at $11.3 \%$ [3]. STDs and unsafe abortions also remain a common public health concern among the youth in Botswana. There has been a concerted effort by both the government and nongovernment organizations to assist adolescents to avoid becoming victims of negative reproductive health problems. Several programs aimed at reducing the frequency and burden of reproductive health problems among the youth have been implemented. For example, an Adolescent Sexual and Reproductive Health (ASRH) Implementation Strategy was developed to guide national and internal players on young people priority needs. One of the core objectives of the strategy was to improve knowledge of ASRH issues which has been achieved. This has not been translated into significant improvements in sexual behaviour among adolescents. However, there have been successes especially in increasing the rate of condom use among adolescents in Botswana [3] but there remain gaps that still need to be filled as some adolescents continue to use condoms inconsistently. Besides abstaining and being faithful to partners, the use of a condom was considered the most viable prevention method among adolescents and as such health education and counselling emphasized the use of the condom by adolescents. Together with non-governmental organizations, the government ensured that condoms are available, affordable and easily accessible for everyone including adolescents. In fact, all contraceptives including condoms are provided free of charge in all public health facilities. There has been a concerted effort to ensure that such commodities are also available and accessible at different places such as public bars, utility service points, public gathering points; just to mention but a few.

Several theories have been developed to explain health seeking behaviour. The most utilized models for health seeking behaviour are the Health Belief Model, the Theory of Reasoned Action (developed later to the Theory of Planned Behaviour), the Pathways Models, the Health Care Utilization or Socio-Behavioural Model by Andersen [4]-[10]. Relationships of variables which are considered relevant for explaining or predicting health seeking behaviours are contained in all these models and this study draws from all of these health seeking behaviour models and borrows some key elements of these theories. The theories recognize the influence of demographic, socioeconomic and psychosocial factors in influencing health seeking behaviour. 
The influence of demographic and socioeconomic factors on condom use has been established [11]-[16]. In addition, the effect of psychosocial factors on condom use has also gained momentum [17] [18]. This study assumes that condom use also depends on psychosocial factors such as perceived severity of the HIV/AIDS threat to health, perceived personal risk of HIV infection, perceived condom attributes, perceived self efficacy and perceived social support. Studies on condom use show that people's perception of risk is associated with high levels of condom use [18]-[23]. Some studies suggest that self efficacy is one of the most important predictors of actual condom use [24] [25] [26]. Perceived condom attributes have also been associated with use of condoms [27] [28]. Perceived social environmental support has also been identified as a major determinant of condom use [29] [30] [31]. The mentioned studies however, indicate that multiple factors determine condom use and that such factors may vary across societies [32] [33].

There are scanty studies that investigated determinants of condom use in Botswana. Such studies focused either on the adult population in general [15] or on a high risk segment of the population such as the military [16] but none on adolescents who constitute a substantial proportion of the population. Other studies on condom use in Botswana focused on awareness, knowledge and practices in relation to condom use among university students [34] [35] [36] and attitudes towards condom use for prevention of HIV infection in Botswana [37]. In some studies carried out in Botswana, the temporal relationships between predictors and condom use have been obscured by the inclusion of condom use variables in the determination of risky sexual behaviour indices [38] [39] [40] [41]. The need to inform sexual and reproductive interventions on factors associated with condom use among adolescents is thus critical as little is known on what influences adolescent condom use in Botswana. The objective of this study is to examine the demographic, socioeconomic and psychosocial predictors of condom use among adolescents aged 15 to 19 years in Botswana. To date, there is little data on factors that influence condom use in Botswana. Information from this study will make policy makers and health program managers understand factors that facilitate or deter condom use among adolescents. This would assist in facilitating the design of effective sexual and reproductive health programs aimed at assisting the youth to avoid reproductive health problems through increased condom use.

\section{Data and Methods}

\subsection{Data Source}

The data source is a cross-sectional population-based Botswana AIDS Impact Survey (BAIS IV) conducted in 2013. The target population for the survey was at two levels; 1) All members of selected households within the ages 6 weeks to 64 years were targeted for HIV testing and 2) All usual members of selected households aged 10 to 64 years were targeted for the behavioural individual questionnaire. A stratified two stage probability sample design was used to select the sample for the survey. The first stage involved selection of enumeration areas (EAs) as primary sampling units (PSUs) using 
probability proportional to number of households in the EA. The second stage involved selecting households using systematic random sampling in each EA chosen during the first stage. Out of a total of 4524 households sampled, $92.9 \%$ responded and the response rate for individuals aged 10 to 64 years within household sampled was $83.9 \%$. The individual questionnaire for the survey covered sexual history and behaviour, male circumcision and sexually transmitted diseases, knowledge about AIDS and level of exposure to interventions, attitudes towards people living with HIV/AIDS, gender issues and counselling, child bearing and antenatal care; and availability of social and medical services. From the 8332 individuals successfully interviewed, 12.6\% (1051) were adolescents aged between 15 and 19 years. Out of the 1051 adolescents that responded, $26.8 \%$ (282) reported to have ever had sex. This study focused on a weighted sample of 221 adolescents (78.4\% of those that have ever had sex) who reported to have been involved in a sexual activity during the 12 months preceding the survey.

\subsection{Measurement of Variables}

\section{- Outcome Variables}

There were two dependent variables for the study 1) Current condom use and 2) Consistent condom use.

Current Condom Use: This dependent variable is a composite index derived from questions on whether an adolescent used a condom the last time they had sex with their most recent sexual partners. The study focused on three most recent sexual partners during the 12 months preceding the survey. For each of the sexual partners, the following question was asked: "The last time you had sexual intercourse with this partner did you or this partner use a condom?" For each affirmative answer, a score of 1 was awarded otherwise a 0 score was given. Summation of scores for each case ranged from 0 to 3. Respondents with a score of 3 were considered to be current condom users and those with 2 or less were considered to be non-users. To ascertain the reliability of variables used to measure current condom use index, a Cronbach's Alpha was estimated as it is good measure of internal consistency. The Cronbach's Alpha was 0.724 which is considered a reliable measure of internal consistency. Any Cronbach's Alpha estimate of 0.7 and above is considered a reliable measure of internal consistency [42].

Consistent Condom Use: The second dependent variable is a composite variable derived from questions on whether adolescents always used a condom with any partner during the past 12 months prior to the survey. For this study, any partner referred to any one of the respondents' last three sexual partners. The following question was asked respondents regarding their recent partners: "In the past 12 months have you always used a condom with this partner?" A score of 1 was awarded to an affirmative answer for each case otherwise a score of 0 was awarded. Summation of scores for each case for this variable ranged from 0 to 3 . Respondents who report to have always used the condom with all of their most recent sexual partners during the 12 months (those with score 3) were considered consistent condom users as this implies that they had a higher propensity to use condoms consistently with all their sexual partners. Those with a 
score of 2 or less were considered to be inconsistent condom users or non-users. The Cronbach's Alpha estimated for construct this dependent variable was 0.791 .

- Independent variables

Independent variables included demographic and socioeconomic factors (age, sex, residence, level of education, employment status, religion, marital status, age at first sex and number of sexual partners last 12 months, HIV/AIDS related knowledge index) and psychosocial factors (perceived severity of the HIV/AIDS threat to health index, perceived personal risk of HIV infection, perceived condom attributes index and perceived self-efficacy index).

\subsection{Operational Definitions of Independent Variables.}

HIV/AIDS related knowledge index. This variable was developed from answers to six questions on knowledge about HIV and AIDS (see Table 1): Correct answers were

Table 1. Percentage distribution of the selected variables depicting positive influence of constructs of behaviour change and HIV/AIDS related knowledge indicators $(\mathrm{N}=221)$.

Respondents Characteristics/Positive Response

\section{Perceived Severity of the HIV/AIDS Threat to Health}

People on ARV should discontinue or stop taking ARVs once they feel better (No)

Acceptable for a man to have more than one sexual partner (No)

Acceptable for a woman to have more than one sexual partner (No)

Perceived Personal Risk to HIV Infection

Personally Concerned about getting HIV since ARV introduction (Yes)

Perceived Condom Attributes

People on ARVs should always use condoms (Yes)

Circumcised males should stop using condoms (No)

Acceptable for a woman to obtain male condoms (Yes)

Acceptable for a man to obtain female condoms (Yes)

A woman has a right to decide if she will have sex (Yes)

Perceived Self Efficacy

Can persuade a sex partner to use a condom (Yes)

Can persuade a sex partner not to have sex if not interested (Yes)

\section{HIV/AIDS Related Knowledge}

Possible for a healthy looking person to have HIV (Yes)

People can reduce their chances of getting HIV/AIDS by using a condom correctly every time they have sex (Yes)

A Person can get infected with HIV through mosquito bites (No)

People can reduce their chances of getting HIV/AIDS by having only one uninfected sex partner who has no other partners (Yes)

A person can get infected with HIV by sharing a meal with a person who has no HIV (No)

People can get HIV because of witchcraft (No)
196

187

190 
awarded a score of 1 otherwise answers were awarded 0 . Summation of all the scores for each case ranged from 0 to 6 . The index was then categorized as low (scores $0-4$ ), medium (score 5) and comprehensive (score 6) knowledge. The Cronbach's Alpha estimated for these variables was 0.738 . A low score implies that the respondent has very poor knowledge on HIV and AIDS related issues whereas medium score implies that the respondent has some knowledge of HIV and AIDS related issues whereas comprehensive indicates thorough knowledge.

Perceived severity of the HIV/AIDS threat to health index. This variable was developed using three questions that determine how adolescents perceive the threat of HIV to health (see Table 1). A positive response reflected positive attitude influencing use of condoms as a way to reduce sexual and reproductive health problems. For each positive answer, a score of 1 was awarded and any other answer was awarded 0 . This also applied to the other three psychosocial factors that follow. Scores for this index ranged from 0 to 3 and were categorised as low (scores 0 - 2) and high (score 3) perception (Cronbach's Alpha $=0.846)$.

Perceived personal risk to HIV infection: Table 1 shows that there was only one question to determine adolescent perception of risk to HIV infection. A positive answer was categorized as high (score 1) and a negative answer as low (score 0 ) perception of risk infection.

Perceived Condom Attributes Index. Five questions were used to determine how adolescents perceive condom attributes (see Table 1). The scores ranged from 0 to 5 and the index was categorized as low (scores 0 - 4) and high (score 5) perception (Cronbach's Alpha $=0.717$ ).

Perceived Self-Efficacy: Only two questions were used to determine self-efficacy index of adolescents in this study (Table 1 ) and maximum score was 2 . The index was categorized as low (scores $0-1$ ) and high (score 2) perception (Cronbach's Alpha $=0.803$ ).

\subsection{Statistical Data Analysis}

Data analysis was performed using the Statistical Package for Social Science (SPSS) version 21. To examine the determinants of condom use, logistic regression was used. A total of eight logistic regression models (four for each dependent variable) were employed to assess the association between independent and dependent variables. For each dependent variable, the first model regressed each independent variable one at a time (univariate); the second model introduced demographic and socioeconomic variables; the third model introduced psychosocial factors only and on the last model (full model), all the independent variables are introduced. Measures of association used are the odds ratio (OR) and the $95 \%$ confidence intervals. To determine whether the models have a better fit, the -2 log likelihood test was used.

\section{Results}

\subsection{Background Characteristics of the Adolescents}

Table 2 shows the frequency distribution of adolescents who reported to have had sex 
Table 2. Percentage distribution of selected demographic, socioeconomic and psychosocial factors of adolescents who had sex during 12 months prior to survey: 2012 BAIS IV.

\begin{tabular}{|c|c|c|}
\hline Background Characteristics & Number & Percentage \\
\hline \multicolumn{3}{|l|}{ Age } \\
\hline Less than 18 years & 40 & 18.1 \\
\hline 18 to 19 years & 181 & 81.9 \\
\hline \multicolumn{3}{|l|}{ Sex } \\
\hline Male & 77 & 34.8 \\
\hline Female & 144 & 65.2 \\
\hline \multicolumn{3}{|l|}{ Residence } \\
\hline Urban & 138 & 62.4 \\
\hline Rural & 83 & 37.6 \\
\hline \multicolumn{3}{|l|}{ Level of Education } \\
\hline Primary or None & 26 & 11.8 \\
\hline Secondary or Higher & 195 & 88.2 \\
\hline \multicolumn{3}{|l|}{ Employment Status } \\
\hline Employed & 38 & 17.2 \\
\hline Unemployed & 183 & 82.8 \\
\hline \multicolumn{3}{|l|}{ Religion } \\
\hline Christian & 184 & 83.3 \\
\hline Other Religion/None & 37 & 16.7 \\
\hline \multicolumn{3}{|l|}{ Marital } \\
\hline Married & 43 & 19.5 \\
\hline Single & 178 & 80.5 \\
\hline \multicolumn{3}{|l|}{ Age at First Sex } \\
\hline Less than 16 years & 38 & 17.2 \\
\hline 16 years or later & 183 & 82.8 \\
\hline \multicolumn{3}{|l|}{ No of Sex Partners Last 12 Months } \\
\hline One Partner & 177 & 80.1 \\
\hline Multiple Partners & 44 & 19.9 \\
\hline \multicolumn{3}{|l|}{ HIV/AIDS Related Knowledge Index } \\
\hline Low & 47 & 22.2 \\
\hline Medium & 64 & 30.2 \\
\hline Comprehensive & 101 & 47.6 \\
\hline \multicolumn{3}{|c|}{ Perceived Severity of HIV/AIDS Threat Index } \\
\hline Low & 33 & 16.8 \\
\hline High & 164 & 83.2 \\
\hline \multicolumn{3}{|c|}{ Perceived Personal Risk to HIV Infection } \\
\hline Low & 94 & 46.3 \\
\hline High & 109 & 53.7 \\
\hline \multicolumn{3}{|c|}{ Perceived Condom Attributes Index } \\
\hline Low & 79 & 38.9 \\
\hline High & 124 & 61.1 \\
\hline \multicolumn{3}{|l|}{ Perceived Self Efficacy Index } \\
\hline Low & 95 & 44.2 \\
\hline High & 120 & 55.8 \\
\hline Total & 221 & 100 \\
\hline
\end{tabular}

during the past 12 months by selected background variables. A total of 221 sexually experienced adolescents reported to have had sex 12 months prior to the survey. A majority of the adolescent were aged between 18 and 19 (81.9\%), were unemployed (82.8\%), 
Christians (83.3\%), single (80.5\%), had secondary or higher education (88.2\%), had sex at 16 years or later $(82.8 \%)$ and had only one sexual partner 12 months prior to the survey $(80.1 \%)$. About two-fifths of the adolescents resided in rural areas and slightly more than a third $(34.8 \%)$ were males.

About half of the adolescents (47.6\%) who had sex during the 12 months prior to the survey had comprehensive knowledge on HIV/AIDS related issues as compared to 22.2\% who had poor knowledge. About four in five adolescent had high perception of the severity of HIV/AIDS threat to health whereas about half (53.7\%) had a high perception of personal risk to HIV infection. Sixty one percent had high perception of condom attributes whereas $55.8 \%$ had high perceived self-efficacy.

\subsection{Current and Consistent Condom Use among Adolescents}

Results show that both current condom use and consistent condom use is high. Almost eight in ten adolescents (78.4\%) reported to have used a condom during the last sexual encounter with most recent partner(s). However, consistency of condom use is lower at $72 \%$ (see Figure 1). Almost all demographic, socioeconomic and psychosocial factors considered were associated with current condom use with most recent partner(s) at bivariate level except for employment status and perceived severity of HIV/AIDS threat index. However, only age at first sex and perceived condom attributes index were not statistically associated with consistent condom use at bivariate level (see Table 3).

\section{Multivariate Logistic Regression Analysis}

\subsection{Current Condom Use}

In the univariate model, current condom use with most recent partner(s) by adoles-

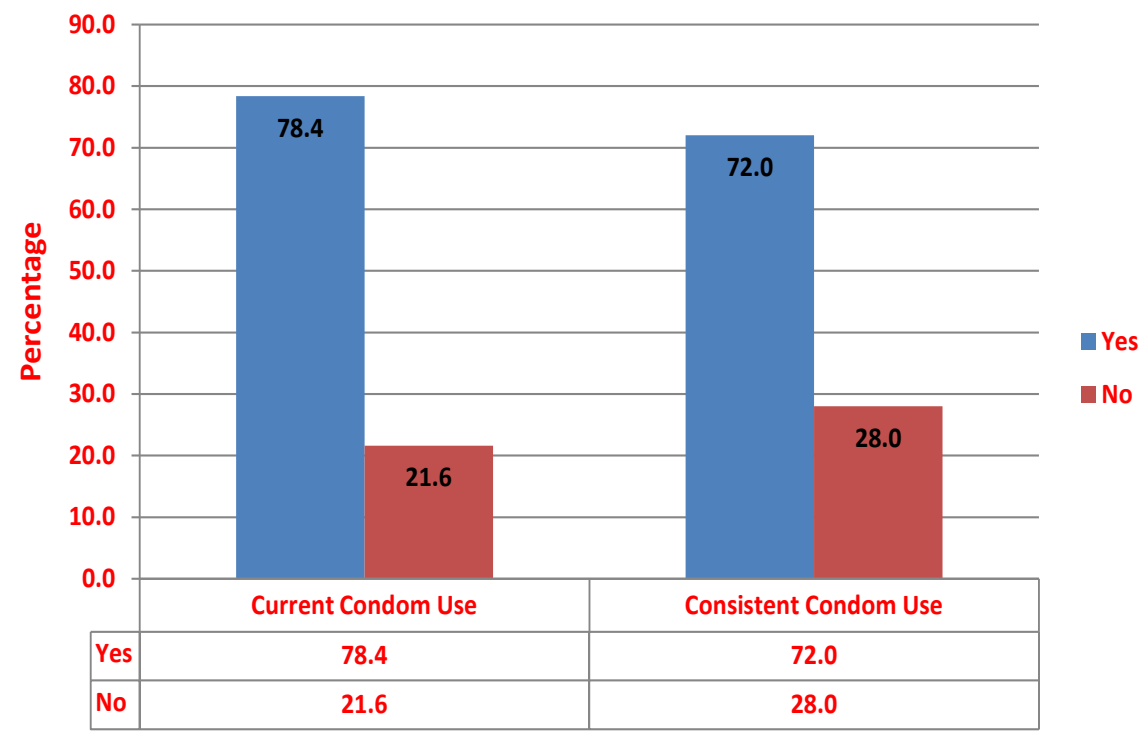

Figure 1. Barchart showing prevalence of adolescent condom use (current condom use) and consistent condom use. 
Table 3. Percentage distribution of adolescents by use of condom during the last sexual encounter with the most recent partner and condom use consistency during last 12 months prior to the survey by selected background characteristics.

\begin{tabular}{|c|c|c|}
\hline & Current Condom Use (\%) $\mathrm{N}=221$ (Weighted) & Consistent Condom Use (\%) $\mathrm{N}=221$ (Weighted) \\
\hline Overall (Total) & 78.4 & 72.0 \\
\hline \multicolumn{3}{|l|}{ Age } \\
\hline 18 to 19 years & 80.6 & 71.4 \\
\hline \multicolumn{3}{|l|}{ Sex } \\
\hline \multicolumn{3}{|l|}{ Residence } \\
\hline Urban & $84.2^{* * *}$ & $75.8^{\star * *}$ \\
\hline Rural & 66.7 & 64.2 \\
\hline \multicolumn{3}{|l|}{ Level of Education } \\
\hline Primary or None & $58.3^{* * *}$ & $50.3^{* * *}$ \\
\hline Unemployed & 78.5 & 73.1 \\
\hline \multicolumn{3}{|l|}{ Religion } \\
\hline Christian & $83.2^{* * *}$ & $77.8^{\star * *}$ \\
\hline Other Religion/None & 59.9 & 49.5 \\
\hline \multicolumn{3}{|l|}{ Marital } \\
\hline Married & $72.4^{* * *}$ & $64.8^{* * *}$ \\
\hline Single & 79.7 & 73.4 \\
\hline \multicolumn{3}{|l|}{ Age at First Sex } \\
\hline Less than 16 years & $75.1^{* * *}$ & 64.8 (NS) \\
\hline Medium & 79.5 & 76.3 \\
\hline Comprehensive & 82.9 & 73.5 \\
\hline \multicolumn{3}{|c|}{ Perceived Severity of HIV/AIDS Threat Index } \\
\hline Low & 80.4 (NS) & $70.3^{* * *}$ \\
\hline High & 80.4 & 74.4 \\
\hline \multicolumn{3}{|l|}{ Perceived Personal Risk } \\
\hline Low & $85.1^{* * *}$ & $77.0^{* * *}$ \\
\hline High & 77.0 & 72.0 \\
\hline \multicolumn{3}{|c|}{ Perceived Condom Attributes Index } \\
\hline Low & $79.1^{\star * *}$ & $74.6(\mathrm{NS})$ \\
\hline High & 81.8 & 74.2 \\
\hline \multicolumn{3}{|c|}{ Perceived Self Efficacy Index } \\
\hline Low & $63.3^{* * *}$ & $60.0^{* * *}$ \\
\hline High & 89.9 & 80.5 \\
\hline
\end{tabular}

\footnotetext{
$* * *=p<0.001$ (Statistically significant); NS $=$ Not statistically significant
} 
cents was found to be positively associated with age, sex, residence, level of education, religion marital status, age at first sex, number of sexual partners last 12 months, perceived personal risk, perceived condom attributes index and perceived self-efficacy. Being an older adolescent (18 to 19 years), having higher level of education (secondary or higher), multiple sexual partners, having had first sex at 16 years or above and being single were associated with higher odds of current condom use with most recent partner(s). Having a high perception of condom attributes and high self-efficacy were also associated with high odds of current condom use with most recent partner(s). The odds of current condom use with most recent partner(s) were lower for adolescents who were female, residing in rural areas, non-Christian and those with high perception of personal risk to HIV infection. The odds of current condom use with most recent partner(s) increased with an increase with the HIV/AIDS related knowledge (see Table 4).

In model 1, all demographic, socioeconomic and HIV/AIDS related knowledge variables were introduced. There were only changes in the direction of association in employment status, marital status, age at first sex, number of sexual partners and HIV/ AIDS related knowledge index. Being unemployed, single, having had sex at 16 years or later, multiple sexual partners and having comprehensive knowledge of HIV/AIDS issues was associated with lower odds of current condom use with most recent partner(s). Although adolescents with medium knowledge had lower odds of being current users of condoms with most recent partner(s) than those with low HIV/AIDS related knowledge, there was no statistical difference between those that had comprehensive knowledge and those with low HIV/AIDS related knowledge.

Model 2 only considered psychosocial factors and only perceived condom attributes index and perceived severity of HIV/AIDS threat to health index were not associated with current condom use with most recent partner(s). Having a high perceived self-efficacy increased odds of being a current condom user with most recent partner(s). However, an adolescent with a high perceived personal risk to HIV infection had lower odds of being a current condom user with most recent partner(s).

The final model included all the variables and the findings indicate that all variables considered were statistically associated with current condom use with most recent partner(s) except for perceived severity of HIV/AIDS threat to health index. Adolescents aged 18 to 19 years were more likely to have used a condom during their last sexual activity as compared to those aged 15 to 17 years (OR, 1.2). Higher odds of current use of condom during last sexual activity with most recent partner(s) by adolescents were associated with higher level of education (secondary or higher (OR, 3.1)). Having high perception of condom attributes and high self-efficacy increased odds of current use of condom with most recent partner(s) (ORs, 1.3; 4.3 respectively). Factors associated with lower odds of current condom use with most recent partner(s) included being female (OR, 0.1), unemployed (0.7), non-Christian (OR, 0.3), single (OR, 0.3), residing in rural areas (OR, 0.7), having had sex at 16 years or above (OR, 0.5$)$, multiple partners (OR, 0.3), having medium $(\mathrm{OR}, 0.4)$ and high perceived personal risk to HIV infection 
Table 4. Logistic regression analysis of factors influencing current use of condom among adolescents in Botswana: 2013 BAIS IV.

\begin{tabular}{|c|c|c|c|c|c|c|c|c|}
\hline \multirow[t]{2}{*}{ Variables } & \multicolumn{2}{|c|}{ Univariate Model } & \multicolumn{2}{|c|}{ Model 1} & \multicolumn{2}{|c|}{ Model 2} & \multicolumn{2}{|c|}{ Full Model } \\
\hline & Odds Ratio & $95 \% \mathrm{CI}$ & Odds Ratio & $95 \% \mathrm{CI}$ & Odds Ratio & $95 \% \mathrm{CI}$ & Odds Ratio & $95 \% \mathrm{CI}$ \\
\hline \multicolumn{9}{|l|}{ Age } \\
\hline Less than 18 years & 1 & & 1 & & & & 1 & \\
\hline 18 to 19 years & $1.767^{\star \star *}$ & $1.659-1.882$ & $1.260^{\star \star \star}$ & $1.160-1.370$ & & & $1.237^{\star * *}$ & $1.126-1.360$ \\
\hline \multicolumn{9}{|l|}{ Sex } \\
\hline Male & 1 & & 1 & & & & 1 & \\
\hline Urban & 1 & & 1 & & & & 1 & \\
\hline Rural & $0.375^{\star * *}$ & $0.354-0.397$ & $0.616^{* * *}$ & $0.574-0.662$ & & & $0.694^{* * *}$ & $0.639-0.753$ \\
\hline \multicolumn{9}{|l|}{ Level of Education } \\
\hline Primary or None & 1 & & 1 & & & & 1 & \\
\hline Secondary or Higher & $3.080^{* * *}$ & $2.862-3.315$ & $2.506^{\star * *}$ & $2.252-2.788$ & & & $3.059^{* * *}$ & $2.689-3.480$ \\
\hline \multicolumn{9}{|l|}{ Employment Status } \\
\hline \multicolumn{9}{|l|}{ Religion } \\
\hline Christian & 1 & & 1 & & & & 1 & \\
\hline Other Religion/None & $0.300^{\star * *}$ & $0.282-0.319$ & $0.276^{\star * *}$ & $0.256-0.298$ & & & $0.322^{* * *}$ & $0.294-0.351$ \\
\hline \multicolumn{9}{|l|}{ Marital } \\
\hline Married & 1 & & 1 & & & & 1 & \\
\hline Single & $1.491^{\star * *}$ & $1.392-1.597$ & $0.548^{\star * *}$ & $0.497-0.604$ & & & $0.339^{* * *}$ & $0.302-0.382$ \\
\hline \multicolumn{9}{|l|}{ Age At First Sex } \\
\hline Less than 16 years & 1 & & 1 & & & & 1 & \\
\hline 16 years or later & $1.256^{\star * *}$ & $1.171-1.347$ & $0.355^{\star \star \star}$ & $0.318-0.397$ & & & $0.495^{\star * \star}$ & $0.440-0.557$ \\
\hline \multicolumn{9}{|c|}{ No of Sex Partners Last 12 Months } \\
\hline One Partner & 1 & & 1 & & & & 1 & \\
\hline Low & 1 & & & & 1 & & 1 & \\
\hline High & $0.948(\mathrm{NS})$ & $0.918-1.083$ & & & $0.957(\mathrm{NS})$ & $0.875-1.045$ & 0.979 (NS) & $0.882-1.088$ \\
\hline \multicolumn{9}{|l|}{ Perceived Personal Risk } \\
\hline Low & 1 & & & & 1 & & 1 & \\
\hline High & $0.583^{* * *}$ & $0.548-0.620$ & & & $0.588^{* * *}$ & $0.551-0.628$ & $0.771^{* * *}$ & $0.717-0.830$ \\
\hline \multicolumn{9}{|c|}{ Perceived Condom Attributes Index } \\
\hline Low & 1 & & & & 1 & & 1 & \\
\hline High & $1.187^{\star \star \star}$ & $1.117-1.261$ & & & $1.122(\mathrm{NS})$ & $1.050-1.200$ & $1.304^{\star * *}$ & $1.164-1.363$ \\
\hline \multicolumn{9}{|c|}{ Perceived Self Efficacy Index } \\
\hline Low & 1 & & & & 1 & & 1 & \\
\hline High & $5.146^{* * *}$ & $4.835-5.478$ & & & $4.693^{\star * *}$ & $4.388-5.021$ & $4.256^{\star * *}$ & $3.940-4.597$ \\
\hline Constant & & & 4.635 & & 0.988 & & 4.187 & \\
\hline Model p-value & & & 0.016 & & 0.029 & & 0.011 & \\
\hline \% Predicted & & & 80.6 & & 80.4 & & 87.0 & \\
\hline -2 Log Likelihood & & & 24368.694 & & 23997.549 & & 20947.960 & \\
\hline
\end{tabular}

*** (asterisk) highlighted in red represent statistically significant. 
(OR, 0.8). Compared to model 1 and model 2, the final had the lowest -2 Log likelihood test indicating a better fit.

\subsection{Consistent Condom Use}

Table 5 shows the logistic regression analysis of factors influencing consistent condom use with most recent partner(s) during the 12 months preceding the survey among adolescents. In the univariate model, consistent condom use with most recent partner(s) was strongly and positively associated with having secondary or higher education, high self-efficacy perception, high perception of severity of HIV/AIDS threat to health, being unemployed, being single, having first sex at age 16 or later and having medium HIV/AIDS related knowledge. Being aged 18 to 19 years, female, non-Christian, rural resident and having a high perception of personal risk to HIV infection was negatively associated with consistent condom use with most recent partner(s). Unexpectedly, number of sexual partners and perceived condom attributes index were not statistically associated with consistent condom use.

Model 2 (multivariate) introduced demographic and socioeconomic factors only. When taking all other demographic and socioeconomic factors into consideration, the direction of the relationship with consistent condom use with most recent partner(s) was modified for residence, marital status, age at first sex and HIV/AIDS related knowledge. Being a rural resident was positively associated with consistent condom use with most recent partner(s) whereas being single, having first sex at 16 years or later and having higher HIV/AIDS related knowledge was negatively associated with consistent condom use with most recent partner(s).

Model 3 considered psychosocial factors only and perceived high self-efficacy, high severity of HIV/AIDS threat to health and high condom attributes was positively associated with consistent condom use with most recent partner(s) among adolescents. Having a high perceived personal risk to HIV infection remained negatively associated with consistent condom use with most recent partner(s).

The final model (full model) introduced all the variables and only age at first sex was not associated with consistent condom use with most recent partner(s). Consistent condom use with most recent partner(s) was positively associated with rural residence $(\mathrm{OR}, 1.4)$, being unemployed (OR, 1.8), having secondary or higher education (OR, $3.2)$, high perception of severity of HIV/AIDS threat to health (OR, 1.2) and high perceived self-efficacy (OR, 2.1). The full model had the lowest-2 Log likelihood test value compared to model 1 and model 2 indicating that it has a better fit with $87 \%$ of the variation explained by the model.

\section{Discussions}

This study set out to investigate the determinants of condom use among adolescents in Botswana. Unlike in other sub-Saharan African countries, condom use by adolescents in Botswana is very high with about eight in ten adolescents reporting having used a 
Table 5. Logistic regression analysis of factors influencing consistency of condom use among adolescents in Botswana: 2013 BAIS IV.

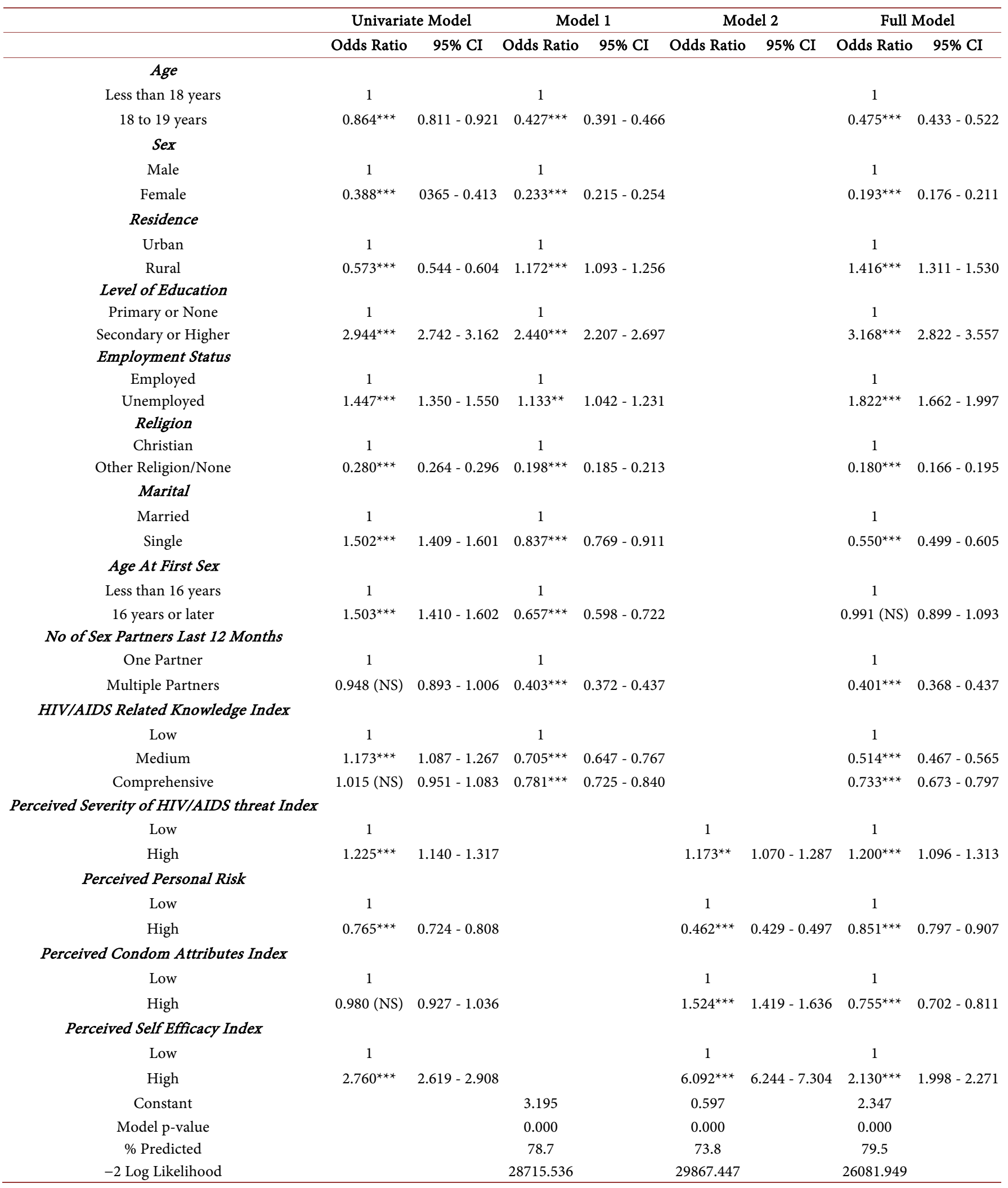

$* * *$ (asterisk) highlighted in red represent statistically significant. 
condom during their last sexual encounter with most recent partner(s). This is indicative of the success of the sexual and reproductive health (SRH) programm interventions put in place through collaborative efforts by both the government of Botswana and Non-Governmental Organizations dealing with adolescent related sexual and reproductive health issues. However, there still remain a huge disparity between current condom use with most recent partner(s) and consistency of use of condoms with most recent partner(s) by adolescents.

This study presented evidence that suggests that just like the demographic and socioeconomic determinants; psychosocial determinants are important drivers of condom use among adolescents. Perception of the severity of HIV/AIDS threat to health was positively associated with higher odds of consistent condom use. Conversely, being more concerned about getting HIV since the introduction of ARV was associated with lower odds of current and consistent condom use. The question on whether one is less or more concerned about getting HIV played a part in measuring the perception on personal risk to HIV infection. This finding was contrary to studies that found a positive association between perception of risk and high levels of condom use among adolescent African adolescents [18]. However, this finding corroborates that of Bauni and Jarabi [21] who suggested that young people often feel invulnerable to HIV infection and that young people usually see AIDS as a distant rather than an immediate threat. Although self-perceived susceptibility to HIV infection may encourage individuals to be able to practice safe sexual behaviours, their ability to initiate and sustain such safe behaviours may largely depend upon societal sexual norms and practices [34].

There are mixed results with regards to the relationship between perceived condom attributes index and use of condom by adolescents. Current condom use was not associated with high perceived condom attributes. On the other hand, the odds of consistent condom use were lower for adolescents with high perceived condom attributes. This finding corroborates the findings by Bauni and Jarabi [21] to some extent who indicated that adolescents see AIDS as a disease that affects other people, and not themselves. Some adolescents might see use of condom as necessary for protection against HIV and STIs only although such risk of infection is regarded as distant rather than immediate by adolescents.

Adolescents with a high perceived self-efficacy had higher odds of being currently and consistent condom users. This finding corroborates the theory of the effect of selfefficacy by Bandura [26]. In addition, self-efficacy has been associated with high levels of condom use [19] [24] [25] [27].

Both use of condom during last sexual encounter and consistency of use of condom differed significantly by age. Although older adolescents were more likely to report using a condom during the last sexual encounter as compared to those aged 17 years or less, they are equally more likely to be inconsistent with use of condom.

Condom use among female adolescents is lower than that of their male counterparts. Findings in this study show that female adolescents were about four times more likely 
to report non-use of a condom during their last sexual encounter with most recent partners. With regard to consistency of condom use during the last 12 months, females were almost five times more likely to have inconsistently used a condom with their most recent sexual partner. A study in Botswana by Marandu and Chamme [37] revealed that men appeared to have a greater tendency to agree with beliefs that encourage non-use of condoms. The likelihood of adolescent women being sexually involved with older men is high, putting adolescent females at a high risk.

Those that had secondary or higher education were almost three times as likely to be current condom users. They were also slightly more than three times as likely to have used a condom consistently as those with primary education or no education. The influence of education attainment on condom use has been widely reported and formal education acts as a preventive factor in Sub-Saharan Africa [43]. It is believed to enhance adolescent's health reasoning and as such adolescents with a higher level of education are likely to adopt safer sexual practices.

Adolescents residing in a rural area were less likely to report having used a condom during their last sexual encounter with most recent partners. Associating lower condom use with staying in rural communities has been reported in other studies and in previous BAIS surveys. However, this study shows a different direction of association with regard to consistency of use. After taking into account other factors, staying in rural areas was associated with a higher level of consistent condom use among adolescents. Another unexpected finding in this study is that the odds of consistent condom use were higher for unemployed adolescents. Also being single was not protective as the odds of using a condom during the last sexual encounter or consistently were lower as compared to being married.

With regard to religion, non-Christian (those belonging to other religions other than Christian, including those with no religious affiliation) had lower odds of use of condom both currently and consistently. Early sexual debut has been associated with short and long term risky sexual behaviours. In this study however, the odds of being a current condom user were lower for those whose age at first sexual encounter was at least 16 years. Age at first sex was however, not statistically associated with consistent condom use.

Multiple sexual partners is regarded as a risky sexual behaviour and has been associated with other risky sexual behaviours. In this study, having multiple sexual partners during the 12 months preceding the survey was associated with lower odds of use of condom during the last sexual encounter with most recent partners and lower odds of consistent condom use among adolescents. This is a disturbing finding which has implications for the SRH and HIV/AIDS programmes. The finding may be related to findings by Bauni and Jarabi [36] who suggested that young people usually see AIDS as a distant rather than an immediate threat. Young people may be knowledgeable about the importance of using a condom consistently or practice safe sex. Unsafe sexual behaviour may be practiced by young people because of circumstances that dictate unsafe sexual behaviour [41]. This dictates that young people should develop life skills to make 
informed choices about safe sex.

Results indicate that having comprehensive knowledge on HIV/AIDS related issues does not necessarily translate to behaviour change. The odds of current and consistent condom use among adolescents lowered with an increase in HIV/AIDS related knowledge index. This mismatch is similar to findings elsewhere [44]. Although knowledge levels are high among adolescents, findings in this study indicate that the Botswana HIV programme needs to look beyond just empowering the nation with knowledge about the epidemic by investigating the influence of other factors.

This study has a few limitations that are worth noting. First, measurement of condom use is based on self reports, a factor that may limit analysis on condom use. Generally, reports on condom use are subject to biases based on social desirability and recall. Respondents may over or under report condom use based on how condom use is stigmatized or how one is expected to behave [45]. Second, the reference period used for questioning respondents on condom use was 12 months (one year) which is a long period to recall all sexual activities experienced. The length of the period may reduce the accuracy of the reports on condom use given by respondent. Third, questions on condom use were limited to three most recent sexual partners and do not take into account condom use for those that had more than three sexual partners during the 12 months preceding the survey. Fourth, the study was limited by the sample size which might have influenced the results and the conclusions. However, the sample was weighted during all analyses.

In conclusion, there is clear evidence of high use of condom among adolescents both as current and consistent users in Botswana. Demographic and socioeconomic factors such as age, sex, residence, education, employment status, religion, age at first sex, number of sexual partners and HIV/AIDS related knowledge are determinants of current condom use. Age at first sex does not influence consistent condom use among adolescents. Psychosocial factors such as perceived severity of HIV/AIDS threat to health, perceived personal risk to HIV infection, perceived condom attributes and perceived self-efficacy influence both current and consistent condom use. These results have sexual and reproductive health programmatic implications. Program interventions working with youth aimed at increasing condom use should not just target demographic and socioeconomic factors, but the psychosocial factors also. It should be noted that the majority of these adolescents in Botswana are either of secondary school going age or have just left secondary education. In Botswana, the secondary school curriculum does provide for sex education which gives adolescents the skills and information they need to refuse sex, or to practice safer sexual behaviour. However, strategies that promote adolescent health such as use of positive youth development programmes may be used to accompany sex education efforts at school level since they provide adolescents with the motivation and confidence needed to use the skills learned during sex education activities [46]. This requires that such programs be promoted as part of the comprehensive approach to promoting adolescent sexual and reproductive health in Botswana. 


\section{References}

[1] Gupta, N. and Mahy, M. (2003) Sexual Initiation among Adolescent Girls and Boys: Trends and Differentials in Sub-Saharan Africa. Archives of Sexual Behavior, 32, 41-53. http://dx.doi.org/10.1023/A:1021841312539

[2] Stover, J., Fidzani, B., Molomo, B.C., Moeti, T. and Musuka, G. (2008) Estimated HIV Trends and Program Effects in Botswana. PLOS ONE, 3, e3729.

http://dx.doi.org/10.1371/journal.pone.0003729

[3] Central Statistics Office (CSO) (2009) 2008 Botswana AIDS Impact Survey III. CSO in Collaboration with the National AIDS Coordinating Agency (NACA), 2009. Gaborone.

[4] Munro, S., Lewin, S., Swart, T. and Volmink, J. (2007) A Review of Health Behaviour Theories: How Useful Are These for Developing Interventions to Promote Long Term Medication Adherence for TB and HIV/AIDS? BMC Public Health, 7, 104. http://dx.doi.org/10.1186/1471-2458-7-104

[5] Noar, S.M. (2007) An Interventionist's Guide to AIDS Behavioural Theories. AIDS Care, 19, 392-402. http://dx.doi.org/10.1080/09540120600708469

[6] Meyer-Weitz, A., Reddy, P., Van Den Borne, H.W., Kok, G. and Pietersen, J. (2000) The Determinants of Health Seeking Behaviour of Adolescents Attending STD Clinics in South Africa. Journal of Adolescence, 23, 741-752. http://dx.doi.org/10.1006/jado.2000.0356

[7] Meyer-Weitz, A., Reddy, P., Van Den Borne, H.W., Kok, G. and Pietersen, J. (2000) Health Care Seeking Behaviour of Patients with Sexually Transmitted Diseases: Determinants of Delay Behaviour. Patient Education and Counseling, 41, 263-274.

http://dx.doi.org/10.1016/S0738-3991(99)00103-2

[8] Rosenstock, I.M., Strecher, V.J. and Becker, M.H. (1988) Social Learning Theory and the Health Belief Model. Health Education Quarterly, 15, 175-183.

http://www.ncbi.nlm.nih.gov/pubmed/3378902

[9] Andersen, R.M. and Newman, J.F. (1973) Societal and Individual Determinants of Medical Care Utilization in the Unites States. The Milbank Memorial Fund Quarterly. Health and Society, 51, 95-124. http://dx.doi.org/10.2307/3349613

[10] Weller, S.C., Ruebush II, T.R. and Klein, R.E. (1997) Predicting Treatment Seeking Behaviour in Guatemala: A Comparison of the Health Services Research and Decision-Theoretic Approaches. Medical Anthropology Quarterly, 11, 224-245.

http://dx.doi.org/10.1525/maq.1997.11.2.224

[11] Nnedu, O.N., McCorvey, S., Campbell-Forrester, S., Chang, J., Salihu, H.M. and Jolly, P.E. (2008) Factors Influencing Condom Use among Sexually Transmitted Infection Clinic Patients in Montego Bay, Jamaica. The Open Reproductive Science Journal, 1, 45-50.

http://dx.doi.org/10.2174/1874255600801010045

[12] Oyediran, K.A., Feyisetan, O.I. and Akpan, T. (2011) Predictors of Condom Use among Young Never Married Males in Nigeria. Journal of Health, Population and Nutrition, 29, 273-285. http://dx.doi.org/10.3329/jhpn.v29i3.7875

[13] Chimbindi, N.E., McGrath, N., Herbst, K., Tint, K.S. and Newell, M. (2010) Socio-Demographic Determinants of Condom Use among Sexually Active Young Adults in Rural KwaZulu-Natal, South Africa. The Open AIDS Journal, 4, 88-95. http://dx.doi.org/10.2174/1874613601004010088

[14] Kankasa, C., Siwale, M., Kasolo, F., Nishiyama, A., Terunuma, H. and Wakasugi, N. (2005) Socioeconomic and Reproductive Factors Associated with Condom Use within and outside of Marriage among Urban Pregnant Women in Zambia. African Journal of Reproductive 
Health, 9, 128-136. http://dx.doi.org/10.2307/3583419

[15] Malema, B.W. (2012) Determinants of Condom Use in Botswana: An Empirical Investigation of the Role of Gender. Botswana Journal of Economics, 10, 59-78.

[16] Tran, B.R., Thomas, A.G., Ditsela, M., Vaida, F., Phetogo, R., Kelapile, D., Chambers, C., Haubrich, R. and Shaffer, R. (2013) Condom Use Behaviours and Correlates of Use in the Botswana Defence Force. International Journal of STD \& AIDS, 24, 883-892. http://dx.doi.org/10.1177/0956462413486889

[17] Katikiro, E. and Njau, B. (2012) Motivating Factors and Psychological Barriers to Condom Use among out of School Youths in Dar es Salaam, Tanzania: A Cross-Sectional Survey Using the Health Belief Model. ISRN AIDS, 2012, Article ID: 170739. http://dx.doi.org/10.5402/2012/170739

[18] Meekers, D. and Klein, M. (2002) Determinants of Condom Use among Young People in Urban Cameroon. Studies in Family Planning, 33, 335-346. http://dx.doi.org/10.1111/j.1728-4465.2002.00335.x

[19] Akwara, P.A., Madise, N.J. and Hinde, A. (2003) Perception of Risk of HIV/AIDS and Sexual Behaviour in Kenya. Journal of Biosocial Science, 35, 385-411. http://dx.doi.org/10.1017/S0021932003003857

[20] Cleland, J. (1995) Risk Perception and Behaviour Change. In: Cleland, J. and Ferry, B., Eds., Sexual Behaviour and AIDS in Developing World, Taylor and Francis, London, 157-192.

[21] Bauni, K.E. and Jarabi, B.O. (2000) Family Planning and Sexual Behaviour in the Era of HIV/AIDS: The Case of Nakuru Distirct in Kenya. Studies in Family Planning, 31, 69-80. http://dx.doi.org/10.1111/j.1728-4465.2000.00069.x

[22] Nzioka, C. (1996) Lay Perceptions of Risk of HIV Infection and the Social Construction of Safer Sex: Some Experiences from Kenya. AIDS Care, 8, 565-579. http://dx.doi.org/10.1080/09540129650125524

[23] Ingham, R. and Holmes, H. (1991) In-Depth of Kenyan KAPB Survey Data. Department of Psychology and Social Statistics, University of Southampton, Southampton.

[24] Baele, J., Dusseldorp, E. and Maes, S. (2001) Condom Use Self Efficacy: Effect on Intended and Actual Condom Use in Adolescents. Journal of Adolescent Health, 28, 421-431. http://dx.doi.org/10.1016/S1054-139X(00)00215-9

[25] Lindberg, C.E. (2000) Knowledge, Self-Efficacy, Coping, and Condom Use among Urban Women. Journal of the Association of Nurses in AIDS Care, 11, 80-90. http://dx.doi.org/10.1016/S1055-3290(06)60387-7

[26] Bandura, A. (1986) Social Foundations of Thought and Action: A Social Cognitive Theory. Prentice Hall, Englewood Cliffs.

[27] Peltzer, K. (2000) Factors Affecting Condom Use among Senior Secondary School Pupils in South Africa. Central African Journal of Medicine, 46, 302-308. http://dx.doi.org/10.4314/cajm.v46i11.8575

[28] Maswanya, E., Moji, K., Horiguchi, I., Nagata, K., Aoyagi, K.I., Honda, S. and Takemoto, T. (1999) Knowledge, Risk Perception of AIDS and Reported Sexual Behaviour among Students in Secondary Schools and Colleges in Tanzania. Health Education Research, 14, 185-196. http://dx.doi.org/10.1093/her/14.2.185

[29] MacPhail, C. and Campbell, C. (2001) "I Think Condoms Are Good but Aai, I Hate Those Things". Condom Use among Adolescents and Young People in a Southern African Township. Social Science \& Medicine, 52, 1613-1628. http://dx.doi.org/10.1016/s0277-9536(00)00272-0 
[30] Gage, A.J. (1998) Sexual Activity and Contraceptive Use: The Components of the Decision Making Process. Studies in Family Planning, 29, 154-166. http://dx.doi.org/10.2307/172156

[31] Wilson, D. and Lavelle, S. (1992) Psychosocial Predictors of Intended Condom Use among Zimbabwean Adolescents. Health Education Research, 7, 55-69.

http://dx.doi.org/10.1093/her/7.1.55

[32] AbdoolKarim, S.S., AbdoolKarim, Q., Preston-White, E. and Sankar, N. (1992) Reasons for Lack of Condom Use among High School Students. South African Medical Journal, 82, 107-110.

[33] Adih, W. and Alexander, C. (1999) Determinants of Condom Use to Prevent HIV Infection among Youth in Ghana. Journal of Adolescent Health, 24, 63-72. http://dx.doi.org/10.1016/S1054-139X(98)00062-7

[34] Hoque, M.E., Ntsipe, T. and Mokgatle-Nthabu, M. (2013) Awareness and Practices of Contraceptive Use among University Students in Botswana. SAHARA J, 10, 83-88. http://dx.doi.org/10.1080/17290376.2013.869649

[35] Hoque, M.E., Ntsipe, T. and Mokgatle-Nthabu, M. (2012) Sexual Practices among University Students in Botswana. Gender \& Behaviour, 10, 4646-4656.

[36] Seloilwe, E., Jack, A., Letshabo, K., Bainame, K., Veskov, D., Mokoto, M., Kobue, M. and Muzila, R. (2001) HIV/AIDS at the University of Botswana: Behavioural and Prevention Issues. Pula: Botswana Journal of African Studies, 15, 204-210.

[37] Marandu, E. and Chamme, M. (2004) Attitudes towards Condom Use for Prevention of HIV Infection in Botswana. Social Behavior and Personality, 32, 491-510. http://dx.doi.org/10.2224/sbp.2004.32.5.491

[38] Letamo, G. and Mokgatlhe, L.L. (2013) Predictors of Risky Sexual Behaviour among Young People in the Era of HIV/AIDS: Evidence from the 2008 Botswana AIDS Impact Survey III. African Journal of Reproductive Health, 17, 169-181.

[39] Modie-Moroka, T. (2009) Intimate Partner Violence and Sexually Risky Behavior in Botswana: Implications for HIV Prevention. Health Care for Women International, 30, 230-231. http://dx.doi.org/10.1080/07399330802662036

[40] Dinkelman, T., Levinsohn, J. and Majelantle, R. (2006) When Knowledge Is Not Enough: HIV/AIDS Information and Risky Sexual Behaviour in Botswana. National Bureau of Economic Research Working Paper 12418, Cambridge.

[41] Letamo, G. (2011) Does Correct Knowledge about HIV and AIDS Lead to Safer Sexual Behaviour? The Case of Young People in Botswana. African Population Studies, 25, 44-62. http://dx.doi.org/10.11564/25-1-266

[42] Cronbach, L.J. (1951) Coefficient Alpha and the Internal Structure of Tests. Psychometrika, 16, 297-334. http://dx.doi.org/10.1007/BF02310555

[43] Baker, D.P., Leon, J. and Collins, J.M. (2011) Facts, Attitudes, and Health Reasoning about HIV and AIDS: Explaining the Education Effect on Condom Use among Adults in SubSaharan Africa. AIDS and Behavior, 15, 1319-1327. http://dx.doi.org/10.1007/s10461-010-9717-9

[44] Mwale, M. (2008) Behavioural Change Vis- $\mathrm{A}-$ Vis HIV/AIDS Knowledge Mismatch among Adolescents: The Case of Some Selected Schools in Zomba. Nordic Journal of African Studies, 17, 288-299.

[45] Curtis, S.L. and Sutherland, E.G. (2004) Measuring Sexual Behaviour in the Era of HIV/AIDS: The Experience of Demographic and Health Surveys and Similar Enquiries. Sexually Transmitted Infections, 80, ii22-ii27. http://dx.doi.org/10.1136/sti.2004.011650 
[46] Gavin, L.E., Catalano, R.F., David-Ferdon, C. and Gloppen, K.M. (2010) A Review of Positive Youth Development Programs That Promote Adolescent Sexual and Reproductive Health. Journal of Adolescent Health, 46, S75-S91.

http://dx.doi.org/10.1016/j.jadohealth.2009.11.215

Submit or recommend next manuscript to SCIRP and we will provide best service for you:

Accepting pre-submission inquiries through Email, Facebook, LinkedIn, Twitter, etc. A wide selection of journals (inclusive of 9 subjects, more than 200 journals)

Providing 24-hour high-quality service

User-friendly online submission system

Fair and swift peer-review system

Efficient typesetting and proofreading procedure

Display of the result of downloads and visits, as well as the number of cited articles

Maximum dissemination of your research work

Submit your manuscript at: http://papersubmission.scirp.org/

Or contact wja@scirp.org 